\section{Cathéters veineux centraux: Évaluation des modalités de rinçage hebdomadaire}

\author{
par Karin Olson
}

\section{Abrégé}

Le Cross Cancer Institute a récemment changé le protocole de soins en matière d'entretien des cathéters veinetx centranx. Il est passé du rinçage quotidien à l'aide de 100 unités d'héparine par $\mathrm{ml}$ dans un soluté physiologique au rinçage hebdomadaire à l'aide de 10 unités d'héparine dans un même soluté, La présente étude avait pour but de déterminer si cette modification du protocole s'accompagnait ou non de variations des tanx d'infection et d'occlusion relatifs aux cathéters veineux centrawx. Les données ont été recueillies auprès de 39 patients (soit 43 lignes de perfusion intraveineuse). Un seul cathéter (2\%) s'est obstrué et le tanx d'infection était de $8 \%$ chez les patients non-candidats à la greffe de moelle osseuse tandis qu'il atteignait $18 \%$ chez les candidats à la greffe de moelle osseuse. L'Institut a utilisé ces résultats pour maintenir le rinçage hebdomadaire dans le cadre du protocole de soins en matière d'entretien des cathéters veineux centratu.

Bien que l'on utilise les cathéters veineux centraux depuis plus d'une décennie, les infirmières ne disposent pas de résultats de recherche sur lesquels baser l'entretien des cathéters veineux centraux. Une de leurs préoccupations majeures concerne la fréquence de rinçage. On est en droit de penser que deux complications - l'infection et l'occlusion peuvent être reliées à la fréquence de rinçage.

\section{Revue des écrits en sciences infirmières Infection}

Les taux d'infection associés aux protocoles de rinçage quotidien des cathéters veineux centraux varient entre 5,6\% (Raaf, 1985) et 46 $\%$ (Al-Sabai, 1987). Il est bien sûr difficile de comparer les données se rapportant aux taux d'infection provenant de différentes études à cause des différences remarquées au niveau de facteurs tels que la définition de l'infection et la nature de la population visée. Toutefois, Weightman, Simpson et Speller (1986) ont proposé qu'une réduction des manipulations quotidiennes pourrait diminuer les infections puisqu'une grande partie des infections associées aux cathéters pouvait être attribuée à la contamination de leur capuchon protecteur.

Dans l'étude effectuée auprès de patients en oncologie, Kelly, Dumenko, McGregor et McHutchion (1990) affirment qu'il y avait infection quand la culture du cathéter ou de son extrémité était positive. Si les cultures se révélaient négatives mais que la condition du patient s'améliorait une fois qu'on avait retiré le cathéter, on classait ce dernier parmi les cathéters infectés. Les auteurs de l'étude ont découvert que, dans le cas d'un rinçage hebdomadaire, $10 \%$ des patients non-candidats à la greffe de moelle osseuse et $30 \%$ des candidats à la greffe de moelle osseuse avaient développé une infection. Ces résultats se comparaient à ceux publiés dans le cadre d'études préalables chez des patients en oncologie où les taux d'infection variaient entre $12 \%$ (Passa, 1985) et $55 \%$ (Al-Sabai, 1987). Toutefois, Kelly et al (1990) ont aussi découvert que le taux d'infection par 100 jours d'insertion était inférieur, et de loin, aux taux déjà signalés pour les protocoles de rinçage quotidien ( $7 \%$ et $25 \%$ respectivement). Quant aux taux d'infection connus pour ce qui est des protocoles de rinçage quotidien, ils allaient de $21 \%$ (Pessa et Howard, 1985) à $53 \%$ (Kappers-Klunne et Degener, 1985). Bien que les études décrites ci-dessus aient certaines limitations du fait des différentes définitions de l'infection et d'autres facteurs, les résultats suggèrent qu'on parviendra à faire baisser le taux d'infection en faisant passer de quotidienne à hebdomadaire la fréquence de manipulation des cathéters veineux centraux. Occlusion

Les écrits actuels contiennent peu d'information sur les rapports qui existent entre le taux d'occlusion et la fréquence de rinçage. Il est difficile de comparer les études réalisées dans ce domaine étant donné que certains chercheurs considéraient qu'une ligne était obstruée si elle ne pouvait pas servir à prélever du sang même si on pouvait la rincer; pour d'autres auteurs, une ligne était obstruée si on ne pouvait la rincer. Petersen, Clift, Hickman et al (1986) ont mené une étude dans laquelle on rinçait les cathéters veineux centraux deux fois par jour avec $6 \mathrm{ml}$ d'héparine dans un soluté physiologique. Ils indiquent qu'on avait dû enlever les cathéters chez $6,7 \%$ des patients à cause de la formation de caillots et d'autres anomalies. Cassidy, Zayko, Bron et al (1987) rapportent la formation d'un manchon de fibrine dans $57 \%$ des cathéters obstrués examinés dans leur étude. Raaf (1985) signale que seulement $3,9 \%$ des lignes de perfusion intraveineuse (sur 698 patients) laissaient passer le liquide perfusé mais qu'elles ne permettaient pas le prélèvement sanguin. Reilly, Steed et Ritler (1984) déclarent que 15 $\%$ des lignes de perfusion intraveineuse examinées dans leur étude ont dû être retirées pour cause d'occlusion. Quant à Kelly et al (1990), ils considèrent qu'une ligne était obstruée s'il leur était impossible de prélever du sang ou de la rincer. Ils rapportent que $10 \%$ des lignes s'obstruaient mais qu'elles redevenaient toutes fonctionnelles par lasuite. Ces résultats suggèrent que le passage d'une fréquence de rinçage quotidienne à une fréquence hebdomadaire n'a pas vraiment d'impact sur le problème de l'occlusion.

C'est ainsi que ces résultats nous ont récemment amenées à changer le protocole de soins en matière d'entretien des cathéters veineux centraux. II est passé du rinçage quotidien à l'aide de $3 \mathrm{ml}$ de salin par la lumière (100:1) au rinçage hebdomadaire à l'aide de $3 \mathrm{ml}$ de salin par la lumière (10:1). Nous consignons dans le présent article les résultats de l'évaluation menée afin de déterminer s'il y a eu altération ultérieure de nos taux d'infection ou d'occlusion relatifs aux cathéters veineux centraux. Nous y étudions également les répercussions que ce changement a sur les coûts.

\section{Méthode}

Ce projet s'intéressait aux problèmes cliniques de l'occlusion et de la septicémie. On y a défini l'occlusion comme étant l'incapacité de rincer et d'obtenir le retour veineux. On a donné la définition suivante au terme "septicémie": une température orale supérieure à $38,5^{\circ} \mathrm{C}$ et des hémocultures positives (le même organisme prélevé sur le cathéter veineux central et sur une veine périphérique). Une patiente présentait une température orale supérieure à $38,5^{\circ} \mathrm{C}$ et une hémoculture négative; elle a dû se faire retirer sa ligne de perfusion à cause d'une infection non contrôlée. Mais sa température est redevenue normale peu après le retrait du cathéter et on a donc compté ce cas parmi les cas de septicémie causée par un cathéter veineux central.

\section{Échantillon}

Notre échantillon se composait de tous les patients ayant reçu un cathéter Quinton Raaf à double ou triple lumière dans notre établissement entre les mois de juin 1991 et mai 1992. On a assuré le suivi des patients tout le long de leur hospitalisation qui, dans le cas de certains patients, incluait aussi des consultations externes.

\section{Collecte des données}

C'est dans une salle d'opération et dans des conditions stériles que tous les cathéters veineux centraux ont été mis en place par des chirurgiens. La consignation aux dossiers a été effectuée par une infirmière en oncologie qui possédait une vaste expérience auprès des candidats à la greffe de moelle osseuse. Les données relatives à l'infection ont été relevées dans les rapports de laboratoire. Étant donné que les médecins doivent rédiger une ordonnance pour les médicaments utilisés en vue de déboucher les lignes obstruées, on a compilé les données relatives à l'occlusion à partir des feuilles d'ordonnance des médecins et des notes d'observation des infirmières. On a vérifié que le nouveau protocole de rinçage hebdomadaire était bien observé par le biais d'entrevues téléphoniques menées auprès d'un 
échantillon de $20 \%$ des participants à l'étude. Ces entrevues étaient effectuées par une étudiante-infirmière du premier cycle.

Résultats

On a recueilli les données auprès de 39 patients (43 lignes). Parmi eux, 25 étaient non-candidats à la greffe de moelle osseuse et 14 étaient candidats à la greffe de moelle osseuse. (Voir le tableau 1.) $61 \%$ des lignes sont restées totalement fonctionnelles. Parmi les lignes restantes, le rinçage se faisait facilement mais il était impossible de prélever du sang dans $23 \%$ d'entre elles. Par la suite, ce problème s'est résolu de lui-même, ce qui signifie que l'extrémité du cathéter reposait temporairement contre la paroi de la veine. Dans $12 \%$ descas restants, le rinçage était facile à faire mais il a fallu recourir à la streptokinase afin de prélever du sang, ce qui indique la présence probable d'un manchon de fibrine à l'extrémité du cathéter. On n'a pas pu rincer une des lignes $(2 \%)$ parce qu'elle était infiltrée. Une seule ligne ( 2 $\%$ ) semblait tout à fait obstruée mais on s'est abstenu d'intervenir puisque son retrait était déjà prévu.

Lorsque le protocole de rinçage quotidien était en vigueur, le personnel infirmier estimait que le taux d'occlusion se situait aux alentours de $10 \%$. On a utilisé un seul échantillonnage proportionnel afin de mettre en évidence la différence existant entre le taux d'occlusion de $10 \%$ observé dans le cadre du rinçage quotidien et celui de $2 \%$ observé dans le cadre du rinçage hebdomadaire. On notait un seuil de signification à 0,038 . Il y a lieu de considérer ces résultats avec prudence étant donné que le taux d'occlusion correspondant au protocole de rinçage quotidien était une estimation. Cependant, il est peu probable que le taux d'occlusion existant dans le cadre d'un rinçage hebdomadaire soit sensiblement pire que dans le cadre d'un rinçage quotidien. Nous croyons que la baisse du taux d'occlusion enregistrée dans le cadre du rinçage hebdomadaire est probablement attribuable à la réduction du nombre de manipulations de la ligne et à sa conséquence naturelle, la réduction des traumatismes de la veine.

Nous ne possédions aucune donnée sur notre propre taux de septicémie relative aux cathéters veineux centraux dans le cadre du rinçage quotidien. Toutefois, comme le tableau 2 le démontre, le taux de septicémie relatif aux cathéters veineux centraux observé dans le cadre du rinçage hebdomadaire chez nos patients non-candidats à la greffe de moelle osseuse - $8 \%$ - est près de celui rapporté par Kelly et al (1990). Nous expliquons ce résultat par le fait que la ligne fait l'objet de moins de manipulations ce qui réduit d'autant les occasions d'introduction de contaminants. Quant au taux d'infection relatif aux cathéters veineux centraux observé chez les candidats à la greffe de moelle osseuse, ilétait de $18 \%$.Ce pourcentage est inférieurà celui obtenu par Kelly et al et nous pensons qu'il résulte probablement de l'emploi dans notre établissement de pansements de gaze plutôt que de pansements occluşifs. Les participants à l'étude menée par Kelly et al portaient des pansements occlusifs sur les sites d'insertion.

Tableau 1: Intégrité fonctionnelle de 43 cathéters veineux centraux rincés une fois par semaine au moyen de $3 \mathrm{ml}$ d'héparine dans un soluté physiologique (10:1)

\begin{tabular}{lrr|} 
Caractéristiques des lignes & N & $\%$ \\
Lignes demeurant fonctionnelles & 26 & 61 \\
$\begin{array}{l}\text { Lignes permettant seulement le rinçage } \\
\quad \text { Problème de position }\end{array}$ & 10 & 23 \\
$\quad$ Streptokinase requise & 5 & 12 \\
Lignes obstruées & 1 & 2 \\
Lignes infiltrées & 1 & 2 \\
\hline
\end{tabular}

Tableau 2: Cas de septicémie dans 43 cathéters veineux centraux rincés une fois par semaine au moyen de $3 \mathrm{ml}$ d'héparine dans un soluté physiologique (10:1)

\begin{tabular}{|c|c|c|c|c|}
\hline Présence & de & cémie & Abse & de septicémie \\
\hline A & $\mathbf{N}$ & $\%$ & $\mathbf{N}$ & $\%$ \\
\hline $\begin{array}{l}\text { Non-candidats à la } \\
\text { greffe de moelle osseuse }\end{array}$ & 3 & 18 & 14 & 82 \\
\hline $\begin{array}{l}\text { Candidats à la greffe } \\
\text { de moelle osseuse }\end{array}$ & 2 & 8 & 24 & 92 \\
\hline
\end{tabular}

Au cours de la seconde phase de l'évaluation, la responsable de la collecte des données a téléphoné à un échantillon aléatoire simple de $20 \%(n-9)$ des patients dont les cathéters veineux centraux avaient été mis en place durant la période couverte par l'étude. Six de ces patients effectuaient le rinçage hebdomadairement. Parmi eux, quatre ne rencontraient aucun problème tandis que les deux autres avaient de la difficulté à démarrer le rinçage mais qu'ensuite, ils étaient capables d'introduire le reste de la solution sans plus de problèmes. Deux des neuf patients ont connu des problèmes de rinçage tels qu'il leur a fallu augmenter la fréquence. Le premier a connu une occlusion pour laquelle on a dû faire appel à la streptokinase. On a ensuite maintenu le circuit en le rinçant tous les deux jours. Le second patient avait du mal à rincer sa ligne au début et en a assuré l'entretien ultérieur en la rinçant tous les deux ou trois jours. Le dernier patient rinçait sa ligne chaque fois que l'on changeait le pansement, simplement par commodité.

Répercussions sur les coûts

D'après les chiffres présentés par Kelly et al (1992), les coûts associés au rinçage quotidien d'une double ligne se montent à environ $8,26 \mathrm{~S}$ par semaine, soit $429,52 \$$ par an. Le rinçage hebdomadaire d'une telle ligne couterait environ $1,18 \$$ par semaine soit $61,36 \$$ par an. Si on considère la durée d'insertion moyenne de 186 jours, les frais de rinçage hebdomadaire se chiffreraient à $31,35 \$(186 / 7 \times 1,18)$ tandis que ceux du rinçage quotidien se monteraient à $219,48 \$(186 / 7 \times 8,26)$, soit une économie de $188,13 \$$ par patient. Comme nous suivons environ 30 patients adultes équipés de nouveaux cathéters veineux centraux, notre établissement économiserait 5644 dollars par an en vertu de ce nouveau protocole.

Conclusion

Compte tenu de ces résultats, on enseigne à tous les patients comment rincer leur(s) cathéter(s) veineux central(ux) de manière hebdomadaire et comment établir une pression positive dans le circuit une fois le rinçage terminé. On conseille à ceux qui éprouvent des problèmes qui ne disparaissent pas instantanément d'en faire le rinçage tous les deux ou trois jours. On mène actuellement une étude afin d'évaluer l'emploi d'un rinçage hebdomadaire réalisé à l'aide d'un soluté bactériostatique.

\section{Remerciements}

L'auteure tient à reconnaître l'aide de Linda Read Paul et de Joanna Pollard qui ont assuré la collecte des données utilisées dans le cadre du projet. Ce projet a été financé par une bourse de perfectionnement au travail du ministère de la Santé de l'Alberta.

\section{Bibliographie}

Al-Sabai, M., Harden, E., Faskin, R., Johnson, G., \& Padmos, A. (1987). The value of prophylactic antibiotics during the insertion of long-term indwelling silastic right atrial catheters in cancer patients. Cancer, 60, 1891-1895.

Cassidy, F., Zayko, A., Bron, K., Reilly, J., Peitzman, A., \& Steed, D. (1987). Noninfectious complications of long-term central venous catheters: Radiologic evaluation and management. American Journal of Radiology, 149, 671-675.

Kappers-Klunne, M. \& Degener, J. (1985). Complications from long-term indwelling central venous catheters, with special reference to infections. Netherland Journal of Medicine, 28, 192-196.

Kelly, C., Dumenko, L., McGregor, E., \& McHutchion, E. (1992). A change in flushing protocols of central venous catheters. Oncology Nursing Forum, 19(4), 599-605.

Pessa, M. \& Howard, R. (1985). Complications of Hickman Broviac catheters. Surgery, Gynecology and Obstetrics, 161, 257-260.

Petersen, F., Clift, R., Hickman, R., Sanders, J., Meyers, J., Kelleher, J., \& Buckner, C. (1986). Hickman catheter complications in marrow transplant recipients. Journal of Parenteral and Enteral Nutrition, 10(1), 58-62.

Raaf, J. (1985). Results from use of 826 venous access devices in cancer patients. Cancer, 55, 1313-1321.

Reilly, J., Steed, D., \& Ritler, P. (1984). Indwelling venous access catheters in patients with acute leukemia. Cancer, 53, 219-223.

Weightman, N., Simpson, L., \& Speller, D. (1986). Source of infection in Hickman catheters. Journal of Clinical Pathology, 39(9), 1046. 